\title{
Applications possibles de la télédétection à l'inventaire et à la caractérisation des ressources en eaux souterraines
}

\author{
Teledetection and groundwater resources
}

\author{
Jacques V. Avias \\ Professeur à l'Université de Montpellier II, \\ Directeur du CERGH et de l'UER*
}

Par deux de ses caractéristiques majeures, à savoir : la possibilité de percevoir à très petite échelle notre planète d'une part, la possibilité de répéter cette perception à des pas de temps réguliers - tous les 9 jours dans les derniers satellites américains E.R.T.S., tous les 2 à 3 jours dans le vaisseau spatial soviétique Météor 2-, beaucoup plus courts avec les aéronefs), la télédétection électromagnétique est en passe de révolutionner les méthodes et les possibilités d'inventaire et de contrôle des ressources naturelles terrestres, d'étude et de caractérisation des grands cycles de matière qui animent la surface du globe :

- cycles biologiques comme le cycle chlorophylien

- cycles inorganiques, tel le cycle de l'eau dont l'importance directe ou indirecte pour l'homme est primordiale.

Dans le cycle de l'eau, l'étude de la partie atmosphérique (hydrométéorologie) et hydrosphérique (hydrologie des eaux de surface : océaniques, côtières, continentales) a, ces dernières années, grâce à la Télédétection, subi une véritable révolution méthodologique et atteint une efficacité jusque là jamais atteinte, notamment pour les prévisions climatologiques et pour les problèmes de courantologie et d'inventaire des états d'interface et de pollution des océans, des fleuves et de l'atmosphère.

Le but du présent exposé est de faire le point sur les applications possibles de la Télédétection à la connaissance de la phase souterraine du cycle de l'eau, c'est-àdire celle qui va de l'infiltration des eaux de pluie aux réémergences naturelles localisées (sources) ou diffuses (zones humides) ou artificielles (puits et forages) en passant par les circulations et les stockages souterrains.

Cette phase souterraine est d'une importance particulière pour l'homme car c'est elle qui permet d'assurer la pérénnité de l'alimentation en eau des populations sans compter divers usages agricoles ou industriels dans toutes les régions où la régularité des précipitations ne permet pas aux eaux de surfaces de suffire aux besoins.

Contrairement à ce qui se passe pour les phases atmosphériques et superficielles, la partie souterraine du cycle de l'eau est beaucoup plus difficile à atteindre par les techniques de la Télédétection, n'étant décelable que par ses effets indirects sur la surface du sol, dans la mesure où l'énergie électromagnétique radiative de cette der. nière, émise et captée, est modifiée par la présence de l'eau souterraine d'une part, est différenciable des autres énergies E.M. émises (liées à l'insolation, à la nature des roches, à la végétation etc.), d'autre part.

Nous allons, dans cet exposé, examiner successivement les différentes techniques applicables et donner quelques exemples tirés de l'expérience que nous avons acquise à Montpellier au G.R.T.M.(**) et au C.E.R.G.H.(***) depuis 1969 avec ou sans l'aide du C.N.E.S. et du C.N.R.S.

Techniques de visualisation directe et techniques photographiques

Nous n'insisterons pas sur la photographie aéroportée, qui jointe à l'observation directe par cet extraordinaire instrument de télédétection qu'est l'œil humain qui s'est au cours des âges géologiques et de l'évolution des

(*) Ressources Naturelles et Aménagement Régional, Place Eugène Bataillon - 34060 Montpellier Cedex - France.

(**) G.R.T.M. : Groupe interdisciplinaire de Recherches sur la Télédétection de Montpellier.

(***) C.E.R.G.H. : Centre d'Etudes et de Recherches Géologiques et Hydrologiques de l'Université de Montpellier II. 
mammifères exactement adapté aux radiations solaires arrivant à la surface du sol par la fenetre majeure de transparence de l'atmosphère, reste et restera fondamentale et particulièrement économique.

Peuvent être décelés la plupart des facteurs conditionnant la présence d'eau souterraine (morphologiques, lithologiques, structuraux, pédologiques) ou trahissant sa présence (végétation) sans compter le repérage direct des exurgences superficielles.

Aux techniques de la photographie classique, perfectionnées par modification des conditions d'angle de prises de vue, par modification des émulsions utilisées, par modification des conditions de développement et de tirage de clichés, sont venues s'ajouter les techniques plus sophistiquées des enregistrements multispectraux.

Dans les techniques de photographie multispectrale (utilisables pour les longueurs d'onde enregistrables par photographie c'est-à-dire allant généralement de 0,30 $\mu \mathrm{m}$ à $1,1 \mu \mathrm{m}$ ), au lieu de capter ensemble toutes les radiations visibles et du proche infrarouge, grâce à un dispositif de filtrage ou de dispersion optique, on enregistre séparément ot simultanément un certain nombre de "bandes spectrales". Les objects terrestres émettant ou réfléchissant différemment, suivant leur nature, les différentes radiations correspondantes, la comparaison des divers clichés obtenus permettra de déceler des différences non perceptibles sur les photographies "globales". Par ailleurs, l'établissement des rapports d'émissivité dans plusieurs bandes d'un même objet permettra souvent d'affiner la discrimination. A citer, à titre d'exemple, l'appareillage multispectral américain Daedalus comportant 10 canaux d'enregistrement ; la camera I $2 S$ particulièrement économique d'utilisation opérant avec 4 bandes grâce à 4 filtres et qui a l'exceptionnel avantage de pouvoir enregistrer simultanément sur un même film standard $24 \times 24$ noir et blanc 4 bandes coloriées dont les enregistrements recombinés permettent la restitution d'une image colorée sur un écran dépoli dans un "lecteur" spécial

A noter que le proche infrarouge, seul susceptible d'être enregistré sur émulsion photographique, a un intérêt spécial : la différenciation des zones humides de surfaces (zones de drainage, zones ayant été atteintes par les crues fluviales, zones d'infiltration, zones d'émergence diffuses, etc.).

\section{Techniques de radiométrie infrarouge}

Ces techniques sont par excellence celles qui fournissent le plus de données pour les problèmes d'eaux souterraines vu l'importance du coefficient thermique de l'eau par rapport à celui des roches. Elles sont basées sur l'enregistrement des radiations infrarouges non enregistrables par émulsions photographiques, grâce à la mise en ouvre de "radiomètres" linéaires ou à balayage, basés sur l'utilisation de cristaux semi-conducteurs spécifiques ("modules"), refroidis par un dispositif cryogénique, qui, frappés par des radiations de longueurs d'onde bien caractérisées donnent naissance à un microcourant qui amplifié et capté sur bande magnétique permet un enregistrement extrêmement précis des variations d'intensité émissive.

Les enregistrements visualisés photographiquement ou sur écran cathodique donnent de véritables cartes des intensités d'émissions radiatives (à $2 / 10$ de degré centigrade près). Les radiations I.R. utilisables sont celles pour lesquelles l'atmosphère normale est transparente, à savoir les radiations I.R. correspondant aux fenêtres de : 1,00 à $1,007 \mu \mathrm{m} ; 1,118$ à $1,23 \mu \mathrm{m}$; 1,58 à $1,70 \mu \mathrm{m} ; 2,18$ à $2,30 \mu \mathrm{m} ; 3,50$ à $4,00 \mu \mathrm{m}$; 9,00 à $11,00 \mu \mathrm{m}$.

En pratique 2 types de radiomètres globaux ou multispectraux sont construits ou utilisés : ceux enregistrant les I.R. dans les bandes 3-5 microns, ceux enregistrant les I.R. dans les bandes 8,5- 14 microns (ex. : en France : appareils "Cyclope" et "Supercyclope" de la S.A.T.E.).

Dans les techniques infrarouges, c'est l'action thermique de l'eau qui sera décelée. Or cette action thermique (et son évolution) dépend de la masse d'eau en jeu, de sa vitesse de circulation et donc de la porosité et de la perméabilité des roches en jeu.

Planche I

Dispositifs d'enregistrement radiométrique (aéroportés ou au sol)

1) Avion monomoteur Marchetti $220 \mathrm{CV}$ équipé de 3 trappes permettant l'utilisation simultanée d'une caméra photographique et de deux radiomètres infrarouge à balayage.

2) Les deux radiomètres I.R. utilisés dans la cabine du Marchetti : au premier plan Cyclope, à l'arrière plan Supercyclope de la S.A.T. (Société Anonyme de Télécommunications, Paris).

3) Exemple de portique (en tube de duralumin) de "vérité terrain" installé au "site du Gour Noir" dans les terrains karstiques Nord montpelliérains par le C.E.R.G.H. On remarquera la plateforme d'enregistrement que l'on peut installer jusqu'à $+12 \mathrm{~m}$.

4) Exemple d'installation radiométrique sur le portique précédent, radiomètre Heiman K.T. 24 (8-14 microns) équipé d'un objectif à faible champ de façon à enregistrer depuis la plateforme à $+12 \mathrm{~m}$ une tache au sol de $2 \mathrm{~m}$ de diamètre (tache de résolution des radiomètres Cyclope et Supercyclope aéroportés). A remarquer également à droite une caméra I.R. AGA.

5) Exemple de visualisation par radiométrie I.R. à balayage (8-12 microns) d'une exurgence d'eau douce karstique en mer (Port Miou-Provence), (d'après E. Henseil et R. Burkaiter, C.E.R.G.H., avril 1974).

6) Exemple de visualisation par radiométrie I.R. à balayage (3-5 microns) d'une faille (faille de Corconne) située entre de calcaires jurassiques (en foncé) et des marnes crétacées (en clair), du Nord montpelliérain, provoquant les deux exurgences du Lirou près du village des Matelles (en noir). On remarquera également les écrans de brumes arrêtant l'émission I.R. dans les thalwegs des calcaires.

7) Exemple dans la même région (zone de Quissac), de la modification d'émission I.R. (plus forte) induite par la circulation souterraine courcircuitant un méandre du Vidourle. 

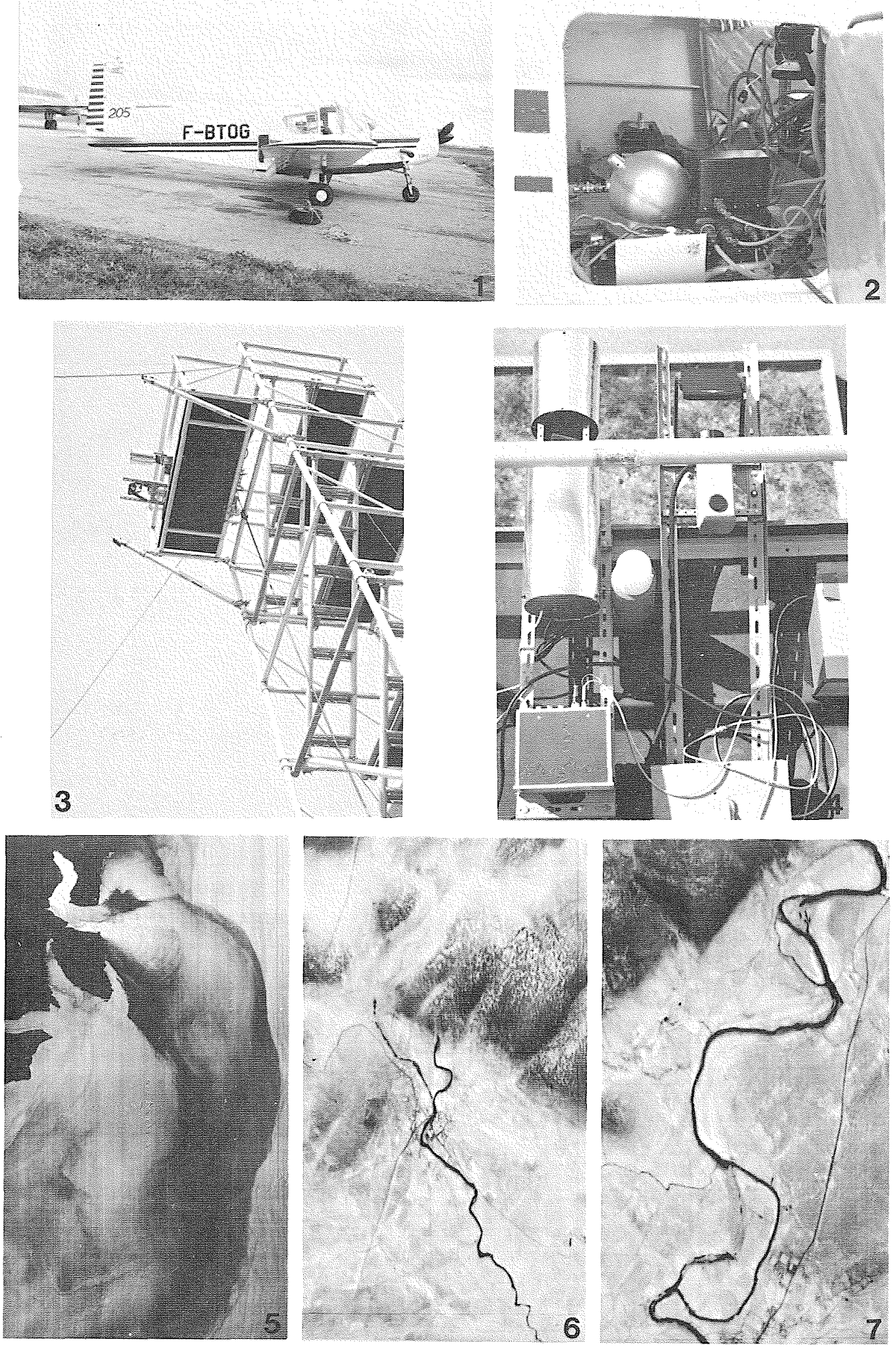


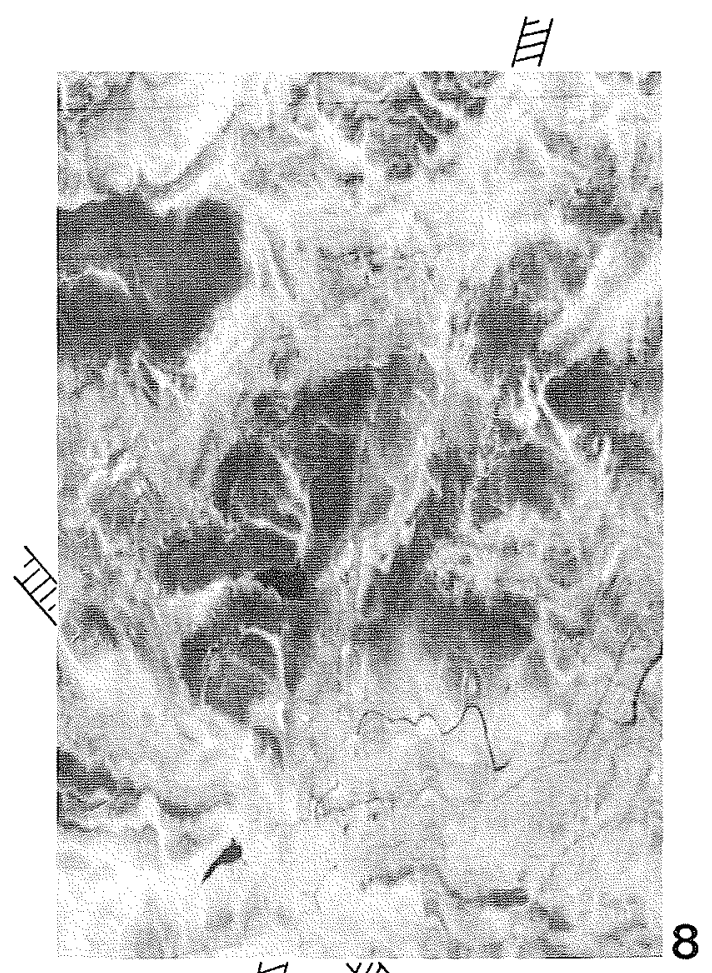

自
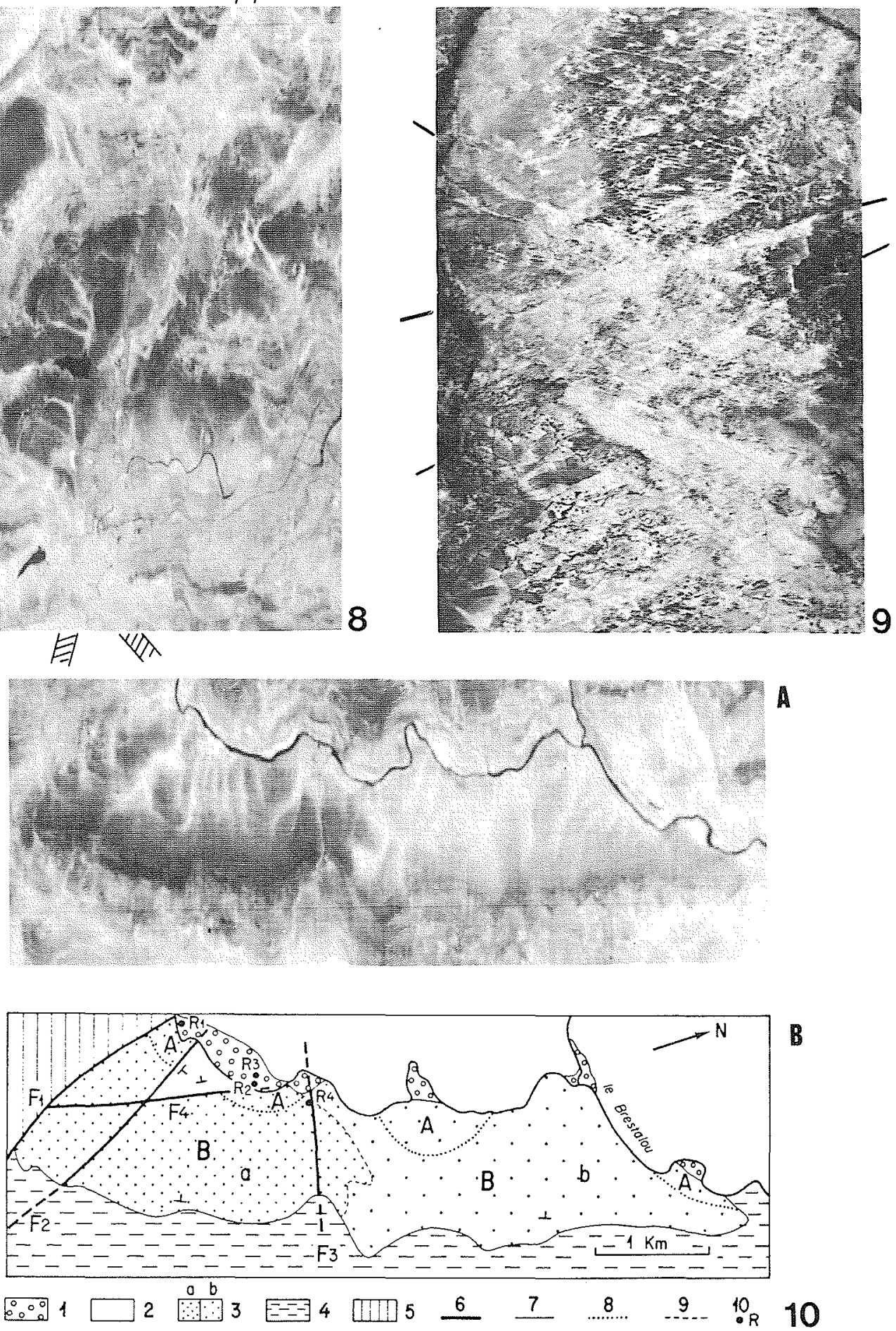

Planche II

8) Mise en évidence de structures faillées non connues (structures drainantes) par radiométrie I.R. (3-5 microns) d'après Marjolet et Salado, C.E.R.G.H., 1972, dans la région de Ceceles (St Mathieu de Tréviers).

9) Délimitation par radiométrie I.R.(3-5 microns) de compartiments à comportement aquifère différent sur le causse de Guillaumard (d'après Burkhalter, C.E.R.G.H., 1972).

10) Délimitation par radiométrie I.R. de fin de nuit et d'hiver (3-5 microns) de la zone tectonisée aquifère (en foncé) dans le monoclinal du Bois de Carnas (Hérault) : A) visualisation en I.R:B) carte géologique : en pointillé serré (3) la zone tectonisée et aquifère avec ses exurgences ou exploitations (R1 à R4). En pointillé lache (1) la zone non tectonisée, non aquifère (d'après J. AVIAS et G. MARJOLET, C.E.R.G.H., 1973). 
Une étude fine des variations thermiques de la surface du sol, en tenant compte des précipitations, de l'heure, des saisons, etc. et si l'on peut éliminer les actions dues aux variations de l'insolation, de l'évapotranspiration, etc. peut permettre de recueillir des renseignements sur ces masses d'eaux souterraines, sur les zones d'infiltration, de circulation, etc. Ceci à la condition que, grâce à des études de "vérité terrain" l'on puisse "étalonner" les indications fournies par la Télédétection en fonction de la nature lithologique, structurale et hydrique des formations en jeu.

Ce problème des "champs thermiques liès aux eaux souterraines" constitue l'un des axes majeurs des recherches entreprises par notre centre de recherche et nous citerons plusieurs études passées ou en cours [1] [2] [4] et notre site test du "Gour Noir" (cf. Pl., 1, fig. 3-4) dans les karsts Nord montpelliérains.

Parmi les possibilités démontrées de la Télédétection infrarouge, nous citerons, à tire d'exemple :

1) La possibilité de localisation des résurgences côtières sous marines d'eau douce (de température différente de l'eau de mer et donc décelables - cf. P1 I, fig. 5);

2) La localisation des zones d'émergence (ou de pertes) diffuses ou localisées (sources) des eaux souter. raines sur terre ou dans les fonds des cours d'eau (cf. P1, fig. 6 et 7 ) ;

3) La localisation de certaines circulations souterraines ou de certains drains notamment le long de zones fracturées ou de failles non perceptibles au sol, par l'effet thermique provoqué en surface par la présence d'eau (cf. P1. I. fig. 7 et P1. II, fig. 8);

4) $\mathrm{La}$ localisation des zones géothermiquement actives ;

5) La localisation de zones d'infiltration préférentielles (ex : zones dolomitiques des Grands Causses);

6) La localisation dans certaines zones favorables et dans certaines conditions d'aquifères (dans la mesure, comme nous l'avons vu, où ces aquifères induisent une modification thermique de l'émission de surface - cf. P1. II, fig. 9 et $10 \mathrm{~A}$ et B).

Il faut insister sur le fait que les interférences thermiques dues à l'insolation, à la végétation ou à la nature des roches, doivent tomber en dessous d'un certain seuil, ce qui signifie que des résultats positifs ne peuvent souvent être obtenus que dans des circonstances spéciales : par ex. l'hiver (réduction de l'effet de la végétation), la nuit (réduction de l'effet induit par l'insolation) et en période sans précipitations et sans vent. Cela suppose que l'on puisse disposer du matériel (porteurs, capteurs, appareillages de mesure au sol) en permanence pour pouvoir profiter des circonstances favorables souvent imprévisibles de façon précise. Cela n'étant généralement pas le cas, il y a un point qui en France paralyse les recherches et les applications en la matière.

\section{Techniques utilisant les ondes millimétriques et centimétriques (microondes et radars)}

Les senseurs correspondants, très sophistiqués, utilisant de façon passive ou active (c'est-à-dire en recueil- lant des "échos" radiométriques d'ondes générées et dirigées artificiellement sur les objets terrestres étudiés), les ondes de longueurs d'ondes millimétriques ou centimétriques (ex : S.L.R. "side looking radar" des américains) sont d'un coût très élevé.

D'un grand intérêt pour certains problèmes météorologiques (localisation des zones d'orages) ou d'hydrologie superficielle (état de la surface de la mer, salinité des eaux, différenciation des neiges, glaces et eaux libres, même à travers des couvertures nuageuses épaisses), ils ne sont pour les eaux souterraines jusqu'à présent que peu importants. Ils permettent seulement, grâce à la pénétration de ces ondes dans le sol, de caractériser l'humidité de certains sols, de déceler certaines structures drainantes de ces sols et dans certaines conditions rares (celles des sols désertiques, sableux, secs) certaines profondeurs de nappes.

\section{Techniques utilisant les lasers}

Nous signalons que ces techniques n'ont d'intérêt qu'indirectement, par exemple en permettant de caractériser l'état de fissuration des formations rocheuses (chaque fissure provoquant une discontinuité de l'écho laser).

\section{Conclusions}

En conclusion, dans le domaine des eaux souterraines, les techniques de la Télédétection (en n'abordant pas ici les techniques d'exploitation des données traitées par ailleurs dans ce colloque) bien que moins prometteuses que dans le domaine de l'hydrométéorologie et de I'Hydrologie superficielle peuvent fournir des renseignements utiles bien que le plus souvent indirects. La Télédétection radiométrique infrarouge à balayage permet cependant dans certaines conditions d'obtenir des résultats intéressants et même parfois spectaculaires.

\section{Bibliographie}

AVIAS J., 1973. - Applications de la Télédétection à l'étude des fluides naturels (in La Télédétection des Ressources terrestres) (Ecole d'Eté. C.N.E.S.-O.N.U., Tarbes 1973), pp. 341-399, 20 fig., 9 tabl, 7 pl. phot. Bibl. Centre National d'Etudes Spatiales, Toulouse.

AVIAS J. et MARJOLET G., 1973. - Note préliminaire sur la possibilité, dans certaines conditions, de détecter la localisation des aquifères fissurés karstiques par Télédétection radiométrique infrarouge aéroportée, d'après un exemple pris dans les karsts nord-montpelliérains de la région du Bois de Carnas (Gard). C.R. Acad. Sc. Paris, t. 276 (8 janvier 1973).

JAMILSON J.A., Mc FEE R.H. et alii, 1963. - Infrared physics and engineering. 673 p., Mc Graw Hill, New York, Toronto, London.

LACAS J.L., 1976. - Introduction à la méthodologie d'étude et d'utilisation des champs hydrothermiques des aquifères karstiques. Mémoires du C.E.R.G.H., t. X, fasc. III, C.E.R.G.A., Montpellier.

NASA et alii, 1972. - Proceedings of an international workshop on earth resources survey systems (1971). Vol. I : Earth resources survey systems, $371 \mathrm{p}$. Scientific and technical information office NASA, Washington. 


\section{Discussion}

Président : M. R. FOULHOUZE

Le Président remercie le Professeur AVIAS de son exposé en remarquant qu'il complète bien celui - précédent - de $M$. VUILlAUME. Comme convenu il propose de joindre les deux mémoires pour la discussion.

M. MAILLARD. - Quel est le coût de la petite station test montée par le Professeur AVIAS?

Professeur AVIAS. - La tour d'observation vaut environ $15000 \mathrm{~F}$. L'ensemble radiomètre et caméra environ $150000 \mathrm{~F}$. En ce qui concerne l'installation de "vérité terrain", elle comprend un forage de $50 \mathrm{~m}$ de profondeur et une série de petits forages à profondeurs variables dans lesquels ont été installées des thermistances, ainsi qu'une station météorologique. L'ensemble représente $40000 \mathrm{~F}$. Au total les frais d'installation à prévoir sont de l'ordre de $250000 \mathrm{~F}$. En ce qui nous concerne, les mesures ont dû être interrompues en raison de l'insuffisance des crédits et de la brièveté des aides de la D.G.R.S.T. accordées pour 18 mois au plus. Or, il faut un an au moins pour acquérir les instruments et monter les expérimentations. Il ne reste que quelques mois pour étalonner les appareils, faire les recherches et le rapport final. Et, par la suite, la D.G.R.S.T. ne donne très généralement d'argent que pour des programmes nouveaux. Il y a là un frein à l'efficacité de la Recherche fondamentale sur lequel je pense qu'il faut insister.

M. LEVY. -- Vous avez parlé de ressources en eau et il s'est agi principalement de présence d'eau. Peut-on obtenir des renseignements sur les volumes ou les débits en jeu?

Professeur AVIAS. - Oui, mais jusqu'à un certain point seulement. $\mathrm{Si}$ on connaît suffisamment l'intensité de l'anomalie thermique, les circulations et les magasins et si l'on peut établir un modèle suffisant, on peut faire un calcul quantitatif approximatif des volumes et des débits en jeu. Mais dans la pratique, la chose est difficile en particulier du fait que l'on n'obtient presque jamais les crédits qui permettraient de déterminer de façon suffisante la structure et le comportement des aquifères ; ce, quel que puisse être l'intérêt économique de cette quantification.
En réponse à une question de M. MUSY sur les observations chiffrées éventuellement enregistrées sur le bassin d'Orgeval (évapotranspiration, rayonnement net), M. VUILLAUME retrace rapidement les circonstances et les procédures associées au premier essai effectué sur le Sénégal en 1975, puis aux opérations rapportées sur le bassin d'Orgeval en 1975 et en octobre 1977

Il indique que - concernant Orgeval - la validité du modèle n'a pas encore pu être vérifiée et que diverses données demandent à être complétées de façon significative.

Il insiste sur la nécessité et les difficultés d'une excellente coordination des diverses équipes et sur les progrès qu'il convenait encore de faire à cet égard. Si les résultats obtenus ne peuvent guère être exprimés en millimètres, ils ne lui paraissent pas moins apporter - en valeurs relatives - des indications intéressantes.

M. COROMPT demande au Professeur AVIAS l'ordre de grandeur de l'épaisseur de roche à travers laquelle on va mettre en évidence la présence d'eau.

Professeur AVIAS. - Cela dépend essentiellement de l'intensité de la fissuration. Dans l'exemple donné, les calcaires fracturés ont une épaisseur d'une centaine de mètres. Les vérifications au sol avec un petit radiomètre à main ont montré qu'à l'aplomb des fissures en communication avec l'aquifère, on pouvait avoir des différences de température de $2^{\circ}$ (de nuit, et en hiver - février -).

\section{COROMPT. - Quelle est l'épaisseur de l'eau ?}

Professeur AVIAS. - La surface de la zone noyée était à 5 ou 6 mètres de la surface du sol à mi-pente. Des exurgences affleuraient par ailleurs en bas de pente, dont plusieurs captées. Le problème dépend seulement de l'influence de la masse d'eau sur la température superficielle de la formation qui la contient et du contraste produit par rapport aux zones non aquifères.

\section{Abstract \\ Possible applications of teledetection for listing and characterizing ground water resources}

By its scanning field and repeatability, electromagnetic teledetection is revolutionizing methods and possibilities of listing and keeping a check on Earth's natural resources. In the case of the water cycle it already has revolutionized wellestablished methods in use in meteorology, for current measurement and detection of pollution. The problem is more difficult in the case of ground water, where water can only be detected indirectly through its effects on the ground surface (temperature variation, effect on vegetation, etc.), and then only if such effects are sufficiently marked to be distinguishable from other electromagnetic energy emissions or absorptions related to lithology, incident sunlight, vegetation, etc. Data recording times have to be selected accordingly. Most of the factors governing or indicating the presence of water in the subsoil can be detected by direct visual observation and conven- tional photography from aircraft; a major step forward has been made with multispectral recording methods whereby closer distinctions can be made between "spectral signatures", especially by establishing the intensity ratios in spectral bands of different wavelengths. The most promising technology, however, seems to be infra-red scanning radiometry (in the 3-5 micron band, or better still, 8.5 - 14 microns - thermography) whereby one can easily distinguish between temperatures differing by as little as $0.2^{\circ} \mathrm{C}$. Owing to its high calorific value, water can appreciably affect the temperature of the rock or air with which it is in contact or of other water mixing with it. As a result of this, it is possible to detect underwater or underground springs, surface springs or losses to rivers, some types of underground drain, preferential infiltration zones, and in some cases (fissured limestone), to locate specific aquifers. 